\title{
Electron diffraction studies of supersonic jets. IV. Conformational cooling of $\boldsymbol{n}$-butane
}

\author{
Richard K. Heenan and Lawrence S. Bartell \\ - Department of Chemistry, University of Michigan, Ann Arbor, Michigan 48109
}

(Received 23 September 1982; accepted 19 October 1982)

\begin{abstract}
Expansions through small tapered nozzles $\left(\sim 10^{-2} \mathrm{~cm}\right.$ inlet diameter) have produced conformational cooling of gas phase $n$-butane to estimated conformational temperatures as low as $180 \mathrm{~K}$. Relaxation into the lower energy trans form was seen with neat butane and with addition of up to $\sim 30 \%$ helium or neon. Thin plate nozzles of comparable diameter do not seem to produce the same effects, presumably because the more rapid cooling they bring about is accompanied by many fewer collisions. Conformational analyses carefully checked for and took into account butane cluster scattering, which if present and ignored, artificially increases the apparent trans mole fraction. At higher concentrations of monatomic carrier gas the cluster scattering becomes strong enough to interfere seriously with the determination of conformational composition. Analysis of the present data and a reanalysis of earlier, conventional, gas electron diffraction data both gave the room temperature trans mole fraction as $64 \%(3 \sigma=9 \%)$ in agreement with a recent spectroscopic inference of $68 \%$.
\end{abstract}

\section{INTRODUCTION}

Gas electron diffraction studies have often been used to determine the distribution among conformations of gas phase molecules and hence to estimate free energy differences between conformers. ${ }^{1,2}$ of particular importance have been studies of the temperature dependence of conformer fractions. ${ }^{2}$ Under the mild expansion conditions of conventional electron diffraction, the conformational temperature of the gas has been shown to be essentially that of the nozzle, ${ }^{3}$ despite significant translational and rotational cooling. We wished to see whether the greater number of collisions experienced by molecules expanding in a supersonic jet would produce conformational cooling of $n$-butane sufficient to be detected by changes in electron diffraction patterns. Cooling has been demonstrated, spectroscopically, by Felder and Günthard ${ }^{3}$ for 1,2-difluoroethane, a molecule with a lower barrier to isomerization than $n$-butane. These workers compared effusive and free jet sample beams in argon matrix deposition, and concluded that gas phase conformations are retained after incorporation into the solid matrix.

After earlier ambiguous studies, the room temperature trans/gauche ratio of gas phase $n$-butane has been derived by Compton et al., ${ }^{5}$ whose analysis of the torsional potential gave $\Delta H_{\mathrm{te}}=3.72 \pm 0.12 \mathrm{~kJ} \mathrm{~mol}^{-1}$ and $68 \%$ trans at $298 \mathrm{~K}$. According to this potential, the trans mole fraction would be expected to increase to about $98 \%$ on cooling to $100 \mathrm{~K}$. To measure conformational temperatures in a butane expansion reliably we must determine changes in conformer population to within a few percent. In the nonequilibrium conditions of a supersonic expansion we cannot simply equate conformational temperature with local vibrational, translational, or rotational temperatures, each of which could be quite different. Even with conventional electron diffraction gas nozzles the translation-rotation temperature may fall hundreds of degrees while leaving the intramolecular vibrational distribution virtually at room temperature. Vibration-translation relaxation of $n$-butane is known to take considerably fewer collisions than required by most other small molecules, ${ }^{6}$ presumably because of its low frequency central torsional vibration. However, this only applies within a single conformational potential minimum, and relaxation of gauche to trans $n$-butane may still require, on average, many more collisions.

Since the results of a previous electron diffraction study ${ }^{7}$ by Bradford, Fitzwater, and Bartell (BFB) appeared not to agree with recent spectroscopic ones, and since we needed a reliable reference trans/gauche ratio, we have reexamined the absolute determination of the trans mole fraction, including the original BFB data as well as our own.

\section{EXPERIMENTAL}

Diffraction patterns were taken of $n$-butane both pure and with added helium or neon. Expansion conditions for particular plates are given in Table I of the preceding paper. ${ }^{8}$ Under certain conditions plates showed little or no evidence of cluster formation, notwithstanding a considerable drop in translational temperature. These plates will be the principal subjects for analyses in this paper. The same effective camera height was used for $n$-butane plates in this work as for benzene plates in a previous study. ${ }^{9}$ This value provided good agreement for $n$-butane and benzene distances in comparison with conventional electron diffraction studies. Further experimental details and data reduction procedures may be found in previous papers of this series. ${ }^{8-10}$

\section{ROOM TEMPERATURE STUDIES}

\section{A. Procedure}

To obtain good starting structure parameters for investigation of conformational cooling we reanalyzed the data of BFB. " Diffraction patterns providing a suitable comparison were taken in the present study using jets derived by passing pure $n$-butane at a moderately high pressure through a thin plate nozzle and skimmer. 
TABLE I. Structural parameters for $n$-butane, ${ }^{2}$ (i) plates $257 / 8$ and $263 / 4$, combined $R^{2}$ and $R^{3}$ sector data, (iii) reanalysis of conventional electron diffraction data of Bradford $e t$ al. (Ref. 7). Uncertainties, $3 \sigma$.

\begin{tabular}{|c|c|c|c|}
\hline & \multicolumn{2}{|c|}{ Experimental } & \multirow{2}{*}{$\begin{array}{c}\text { Calculated } \\
\text { Ref. } 7\end{array}$} \\
\hline & (i) This work ${ }^{b}$ & (ii) $\mathrm{BFB}$ & \\
\hline Nozzle & Thin plate ${ }^{c}$ & Tubular & $\cdots$ \\
\hline Diameter (mm) & 0.10 & 0.28 & $\cdots$ \\
\hline Reservoir (psia) & 25 & 0.6 & $\cdots$ \\
\hline$r(\mathrm{C}-\mathrm{C})$ & $1.531(2)$ & $1.531(1)$ & $\cdots$ \\
\hline$r(\mathrm{C}-\mathrm{H})$ & $1.119(2)$ & $1.119(2)$ & $\cdots$ \\
\hline$\angle(\mathrm{CCC})$ & $113.3(4)$ & $113.7(3)$ & $\cdots$ \\
\hline $\begin{array}{l}\angle(\mathrm{CCH})_{\mathrm{av}} \\
\text { (gauche }\end{array}$ & $110.7(4)$ & $110.8(4)$ & $\cdots$ \\
\hline dihedral) & $72.4(48)$ & $70.5(45)$ & $\cdots$ \\
\hline$l(\mathrm{C}-\mathrm{C})$ & $0.054(3)$ & $0.054(2)$ & 0.051 \\
\hline$l(\mathrm{C}-\mathrm{H})$ & $0.088(3)$ & $0.083(2)$ & 0.079 \\
\hline$l(\mathrm{C} \cdots \mathrm{C})_{1,3}$ & $0.068(6)$ & $0.070(4)$ & 0.072 \\
\hline$l(\mathrm{C} \cdots \mathrm{H})_{1,3}$ & $0.111(4)$ & $0.111(4)$ & 0.109 \\
\hline$l(\mathrm{C} \cdots \mathrm{C})_{1,4, \text { sanche }}$ & 0.185 (fixed) & 0.185 (fixed) & 0.185 \\
\hline$l(\mathrm{C} \cdots \mathrm{C})_{1,4, \text { trans }}$ & 0.072 (fixed) & 0.072 (fixed) & 0.072 \\
\hline$\%$ trans & $64(7)$ & $64(9)$ & $\ldots$ \\
\hline$\left(\sigma_{L} / L\right)^{d}$ & 0.008 (fixed) & 0.0 (fixed) & $\cdots$ \\
\hline
\end{tabular}

Distances $r_{g}$ and amplitudes of vibration $l_{g}$ in $\AA$. Angles in degrees. All represent averages over trans and gauche conformers. Amplitudes not given were fixed at calculated values of Ref. 7. Uncertainties in parentheses represent three standard deviations and do not include any estimates for systematic error.

${ }^{b}$ Camera height calibrated by comparison with previous benzene and $n$-butane results.

'Used with skimmer.

${ }^{d} \sigma_{L} / L$ represents ( $\mathrm{rms}$ breadth of gas jet)/(camera height), an experimental parameter strongly correlated with vibrational amplitudes; see Ref. 10.

After compensation for the effects of the considerably broader gas jet in the present experiments $\left(\sigma_{L} / L\right.$ $=0.008$, see Ref. 10) the two sets of structural parameters (Table I) converged to the same values within experimental error. Vibrational amplitudes for the analyses of Table I were fixed at their calculated room temperature values, apart from those for bonded, and 1, 3 nonbonded distances, as listed in the table.

An absolute determination of the trans/gauche population ratio by electron diffraction for a molecule such as $n$-butane encounters certain complications. Scattered intensities change little because only the longer nonbonded distances alter significantly, as shown by the calculated intensity difference curve in Fig. 2 of the preceding paper. ${ }^{8}$ Apparent trans mole fractions are correlated with the gauche CCCC dihedral angle, and the $l(\mathrm{C} \cdots \mathrm{C})_{1,4}$ gauche and trans amplitudes of vibration. Calculated values ${ }^{7}$ of $l(\mathrm{C} \cdots \mathrm{C})_{1,4}$ amplitudes are 0.185 and $0.072 \AA$, respectively for gauche and trans conformations at $300 \mathrm{~K}$. Starting with the BFB fit of Table $I$, if we released the trans 1,4 amplitude it refined to $0.073(2) \AA$ with an increase in trans mole fraction of $1.4 \%$. Similarly, if we released just the gauche 1, 4 amplitude (as was done by BFB), it fell to below $0.073 \AA$ with an increase in trans mole fraction to above $74 \%$. However, reducing both the gauche and trans 1, 4 amplitudes to their approximate calculated values at $100 \mathrm{~K}, 0.115$ and $0.065 \AA$, respectively, increased the trans mole fraction by only $0.8 \%$. Both amplitudes are correlated with the mole fraction, but in opposite directions, so that consistent values, as should occur with vibrational cooling, have little effect on the apparent mole fraction. Our calculated 1, 4 amplitudes are unlikely to be grossly in error since the torsional frequency calculated by the BFB force field $\left(-102 \mathrm{~cm}^{-1}\right)$ was reasonably close to the recently observed value $(\sim 116$ $\left.\mathrm{cm}^{-1}\right) .^{5}$

\section{B. Results}

As shown in Table I, structural and conformational results deduced from intensities obtained with our thin plate nozzle (Fig. 1) are in excellent agreement with those found in a reanalysis (Fig. 2) of the BFB data. Moreover, contrary to the original BFB refinement, the present determinations of the conformational composition yielding $64 \%(\sigma=3 \%)$ trans, including or excluding BFB data, are consistent with the spectroscopic inference of $68 \%$ at $298 \mathrm{~K}$. We are unable to account for the low mole fraction of trans conformer $53.5(9.0) \%$ reported by BFB. Perhaps the discrepancy can be attributed in part to the different nonbonded asymmetry constants adopted and to the less direct procedure applied by BFB who carried out a series of refinements each at fixed conformational composition, instead of a simultaneous refinement of structure and composition. While it is reassuring that our reference room temperature trans mole fraction, for comparison with results from conformationally colder jets, is close to the spectroscopic value, certain questions are raised. We have assumed in Table I that data from plates 257-264 correspond to room temperature when in fact we used a pressure of 25 psia through a thin plate nozzle of diameter $0.1 \mathrm{~mm}$. Such conditions are expected to produce considerable translational cooling. We can understand the result if we hypothesize that the expansion was sufficiently fast to freeze in the room temperature conformational population.

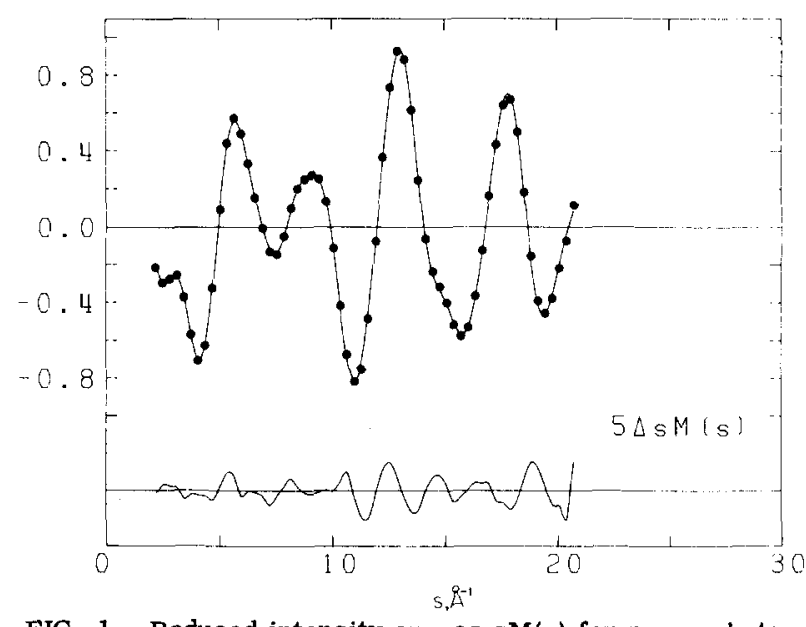

FIG. 1. Reduced intensity curves $\mathrm{sM}(s)$ for pure $n$-butane, 25 psia, taken with a thin plate nozzle: Combined data from plates $257 / 8$ and 263/4. Points are experimental data, solid line is theoretical and the lower trace is five times the difference between them. 


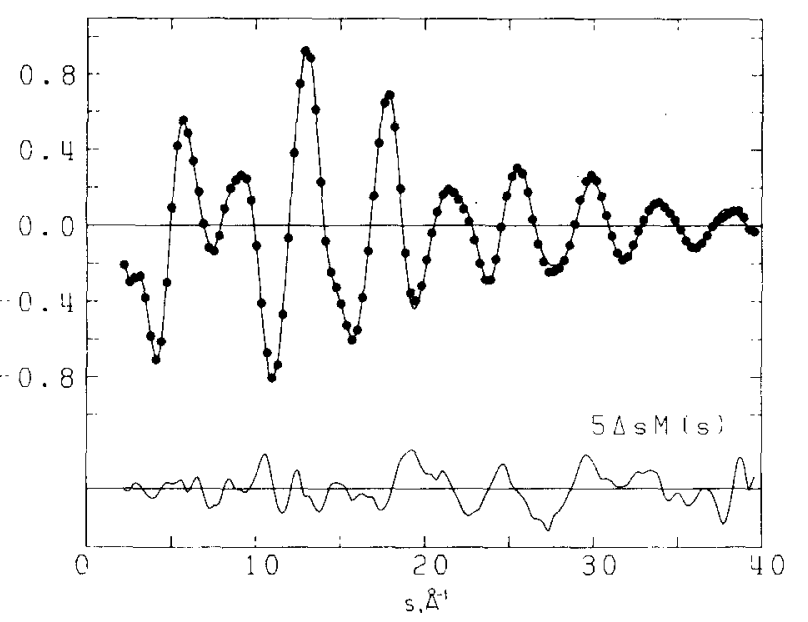

FIG. 2. Reduced intensity curves $\mathrm{sM}(s)$ for a reanalysis of the data of Bradford et al. (Ref. 7); see Table I.

\section{CONFORMATIONAL COOLING}

\section{A. Test of influence of data range}

In most of our diffraction experiments pictures were only taken with a single rotating sector and one camera height, restricting the $s$ range of the available data. It was important, therefore, with the room temperature data discussed above, to investigate the effect of different data ranges on the apparent conformer population. A few results are presented in Table II. In each case structural parameters were as for plates 257-264 in Table I except that the $\mathrm{C}-\mathrm{C}$ distance, CCC angle, conformer fractions, and background function were allowed to vary. (Refining fewer parameters gave slightly lower error estimates, according to normal error theory, than in Table I.) Data from our $R^{3}$ sector alone, plates 257 and 258, gave a high value of the trans fraction, with the largest error. Taking only $R^{2}$ data, plates $263 / 4$, also gave a higher value than from combined $R^{2}$ and $R^{3}$ data. There seems to be some slight, systematic difference between the data from the two sectors. The information in Table II provided a basis to look for changes in conformer fraction when we only have data from a single sector. Tests in which both $R^{2}$ and $R^{3}$ rotating sectors were used with carefully reproduced conditions show reasonable agreement for changes in conformer fractions, irrespective of the sector or sector combination employed.

\section{B. Compensation for systematic errors}

Differences between observed and calculated $M(s)$ curves, such as for the room temperature plates described in this section, represent to a large extent systematic error. In particular, errors arise from the simplified expressions of standard scattering theory, as has been demonstrated for $\mathrm{SF}_{6} .^{11,12}$ Systematic errors, when treated as random errors in normal error theory, suggest larger standard deviations for structural parameters than the precision of electron diffraction data would imply. By subtracting a "systematic residual" and repeating the structural analy sis some idea of the genuine least-squares precision may be obtained. It is plausible to assume that systematic residuals depend little upon the conformational ratio. If we adopt this assumption, results indicate that we have the sensitivity to determine changes in conformer ratio accurately enough to observe conformational cooling.

In results described in the following, we resorted to subtraction of the obs-calc residual of our "control plates" 257/8 and/or 263/4 from other plates. Apparent standard errors of the conformer fraction were thereby reduced by a factor of up to 2 , though the improvement varied from plate to plate.

\section{Discrimination between cluster scattering and conformational cooling}

As explained in the previous paper, the presence of cluster scattering confuses a determination of conformer fractions for $n$-butane, unless the cluster contribution is allowed for explicitly. A procedure to do this is outlined in the following. Intensity data from plate 369 were used as a standard cluster signal as described below.

Reference structure and adjustable parameters were as for Table II discussed in the previous section. In the first place the scale factor for a plate taken with He or Ne was adjusted ${ }^{10}$ where appropriate. (Note that plates 332 and 334 were for pure $n$-butane.) Contrary to our normal practice for gas mixtures, monatomic carrier contribution was then actually subtracted from the leveled intensity. In place of the carrier gas intensity we introduced the cluster scattering from plate 369. A cluster scale factor and other refining parameters were then optimized, enabling the determination of a cluster contribution for any given plate relative to plate 369. Two "cluster intensity" curves were prepared in slightly different ways for analyses labeled A and $B$ in Table III. In both cases the control plates 263/ 4 residual was first subtracted from plate 369 . Since the average trans mole fraction of monomer in the jet was not known, plate 369 was refined with both a $100 \%$ trans model (analysis A) and a 68\% trans model (analy sis $B$ ), as two extreme cases. A variety of cases were investigated, some showing strong and some weak cluster formation. Since systematic residuals have been subtracted in each case the least-squares errors for the conformer fractions need to be combined with analogous

TABLE II. Apparent trans $n$-butane mole fractions, determined from different ranges of diffraction data, used as a basis for comparison of conformational temperatures in Table III. ${ }^{2}$

\begin{tabular}{|c|c|c|c|c|}
\hline Plates & $\begin{array}{l}\text { Degree of } \\
\text { sector opening } \\
R^{n}\end{array}$ & $s_{\min }$ & $s_{\max }$ & $\begin{array}{l}\% \text { trans } \\
( \pm 3 \sigma)\end{array}$ \\
\hline $257 / 8+263 / 4$ & $2+3$ & 2.2 & 21.0 & $64(7)$ \\
\hline $263 / 4$ & 2 & 2.2 & 11.9 & $71(11)$ \\
\hline $257 / 8$ & 3 & 3.8 & 21.0 & $77(15)$ \\
\hline
\end{tabular}

2Only the $\mathrm{C}-\mathrm{C}$ distance, $\mathrm{CCC}$ angle, background function, and trans mole fraction were varied. All other parameters as in Table I, Column (i). 
TABLE III. Cluster contribution and trans $n$-butane mole fraction for expansions of pure butane, butane plus helium, and butane plus neon (see Ref. 8). Cluster fractions are relative to plate 369 from which two, slightly different, cluster patterns were extracted for analyses $A$ and $B$ (see the text). Conformational temperatures are estimated from changes in conformer fraction. Uncertainties, $3 \sigma$.

\begin{tabular}{|c|c|c|c|c|c|c|c|c|c|}
\hline \multirow[b]{2}{*}{ Plate } & \multirow[b]{2}{*}{$\begin{array}{l}\text { Rotating } \\
\text { sector } \\
\text { radius }\end{array}$} & \multirow[b]{2}{*}{$\begin{array}{l}\text { Nozzle }^{\mathrm{a}} \\
\text { diameter } \\
(\mathrm{mm})\end{array}$} & \multirow[b]{2}{*}{$\begin{array}{l}\text { Total } \\
\text { pressure } \\
\text { (psia) }\end{array}$} & \multirow[b]{2}{*}{$\begin{array}{l}n \text {-butane } \\
\text { pressure } \\
\text { (psia) }\end{array}$} & \multicolumn{3}{|c|}{ Analysis A } & \multicolumn{2}{|c|}{ Analysis B } \\
\hline & & & & & $\begin{array}{l}\text { Cluster } \\
\text { fraction }\end{array}$ & $\%$ trans & $\begin{array}{l}\text { Conf. } \\
\text { temp. } \\
\text { (K) }\end{array}$ & $\begin{array}{l}\text { Cluster } \\
\text { fraction }\end{array}$ & $\%$ trans \\
\hline 369 & 2 & 0.275 & $20 \mathrm{Ne}$ & $\sim 5$ & $(1.0)^{b}$ & $(100.0)^{\mathrm{c}}$ & $\cdots$ & $(1,0)^{\mathrm{b}}$ & $(68.0)^{c}$ \\
\hline $267 / 8$ & 2 & 0.12 & $70 \mathrm{He}$ & 15 & $0.32(13)$ & $81(13)$ & $\cdots$ & $\cdots$ & $\cdots$ \\
\hline $314 / 5$ & 2 & 0.145 & $30 \mathrm{He}$ & 17 & $0.49(75)$ & $92(23)^{d}$ & $\cdots$ & $\cdots$ & . \\
\hline 322 & 2 & 0.145 & $50 \mathrm{He}$ & 15 & $0.59(8)$ & $91(8)$ & $\cdots$ & $0.58(8)$ & $75(9)$ \\
\hline 332 & 2 & 0.275 & Pure & 25 & $0.00(5)$ & $86(6)$ & 190 & $\ldots$ & $\ldots$ \\
\hline 334 & 2 & 0.275 & Pure & 15 & $0.01(4)$ & $84(6)$ & 205 & $\cdots$ & $\cdots$ \\
\hline $364 / 5$ & 2 & 0.145 & $20 \mathrm{Ne}$ & 15 & $0.29(6)$ & $88(7)$ & $\cdots$ & $0.29(6)$ & $80(7)$ \\
\hline 402 & 2 & 0.296 & $20 \mathrm{He}$ & 15 & $0.02(6)$ & $88(9)$ & 180 & $0.03(6)$ & $87(10)$ \\
\hline $466-469$ & 2 & 0.240 & $30 \mathrm{He}$ & 20 & $0.07(6)$ & $84(11)$ & 205 & $\cdots$ & $\cdots$ \\
\hline $466-474$ & $2+3$ & 0.240 & $30 \mathrm{He}$ & 20 & $0.07(6)$ & $74(7)$ & 220 & - & $\cdots$ \\
\hline $477-479$ & 2 & 0.240 & $25 \mathrm{He}$ & 21.3 & $0.04(6)$ & $78(9)$ & 245 & $0.04(6)$ & $78(7)$ \\
\hline $477-484$ & $2+3$ & 0.240 & $25 \mathrm{He}$ & 21.3 & $0.04(5)$ & $70(5)$ & 250 & .. & .. \\
\hline
\end{tabular}

${ }^{2}$ Further experimental details in Table I of preceding paper, Ref. 8. All are tapered glass nozzles, except for $267 / 8$, which used a tubular nozzle.

butane largely condensed into clusters. Cluster intensity adopted as reference.

estimates for the control plates 257-264 to give an error estimate for the change in conformer fraction. In fact the errors in Table III dominate, probably due to the uncertainties associated with the cluster contribution. One case, plates $314 / 5$, was included where the cluster component was appreciably different from that of plate 369 as illustrated in the previous paper. This gave much larger errors and a considerable residual compared to say, plates 332 or 402 (see Fig. 3). As

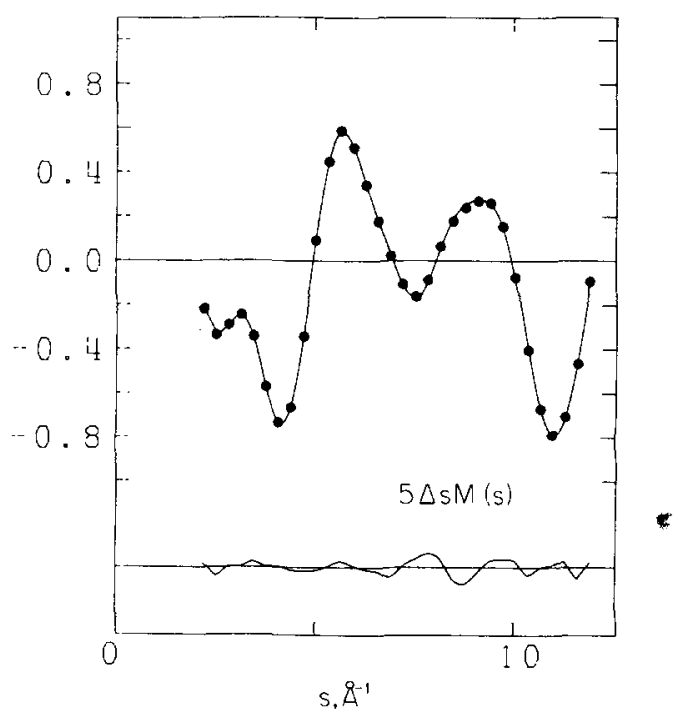

FIG. 3. Reduced intensity curves $s M(s)$ for plate 332 , pure $n$ butane (reservoir pressure 25 psia, tapered nozzle) following analysis $A$ of Table III, systematic residual removed. Corresponding $\mathrm{sM}(s)$ curves for plates with carrier gas are indistinguishable when corrected for cluster scattering and plotted on this scale unless the carrier pressure is high enough to induce strong clustering.
${ }^{c}$ Input assumption distinguishing analyses $\mathrm{A}$ and $\mathrm{B}$. Of consequence only when substantial cluster formation accompanies cooling.

${ }^{d}$ Poor fit due to different shape of cluster contribution; see Ref. 8.

with room temperature data, it seems that our simplifying assumption of room temperature amplitudes of vibration for the important $1,4 \mathrm{C} \cdots \mathrm{C}$ distances has fortuitously little effect. For plate 332, decreasing these two amplitudes to $100 \mathrm{~K}$ values increased the trans fraction from $85.5 \%$ to $86.1 \%$ and gave a significantly worse squared error sum.

\section{Results}

Included in Table III are results for plates showing both strong and weak cluster formation. The determined cluster fractions compare well with corresponding residual heights in Table I of the previous paper. Where the cluster fraction is small, the trans mole fraction is almost the same for analysis $A$ and $B$, and conformational cooling is seen. By relating the changes in trans mole fraction between Tables II and III to changes from $68 \%$ at room temperature, we estimate conformational temperatures at least as low as $180 \mathrm{~K}$. Final residuals are illustrated by plate 332 for pure $n$-butane in Fig. 3 and are pleasingly small. Corresponding residuals for plates taken with carrier gas closely resemble those of Fig. 3 , in amplitude and features, unless the carrier pressure is high enough to induce strong clustering. Indeed, when similar nozzles are used, the resemblance is striking enough to indicate that systematic residuals for conical nozzles are slightly different from those for thin plate nozzles (which, as described above, were adopted in systematic corrections).

The analysis in Table III assumed that the cluster intensity from plate 369 remains good over a wide range of expansion conditions. This is a plausible simplification to render analyses tractable. In a liquid, as this appears to be, the average nearest neighbor interac- 
tions which dominate the scattering are unlikely to change much with cluster size. However, changes in cluster temperature should strongly affect the rate of damping of the cluster intensity as shown by the theoretícal intensities of the previous paper. ${ }^{8}$ The differences in trans fraction between analyses $\mathrm{A}$ and $\mathrm{B}$ are almost exactly, as expected, consistent with an imposition of the limits of $100 \%$ and $68 \%$ trans, respectively, on reaching the same size cluster signal as displayed by plate 369 . (Each difference should be $32 \% \times$ cluster fraction.) By assuming a conformational ratio for the experimental cluster signal one may estimate the conformational ratio of the "free" $n$-butane for plates such as 322 or $364 / 5$. Because of the additional assumptions needed, conclusions become speculative and temperature estimates are not given in these cases.

\section{v. DISCUSSION}

Cooling produced by expansion of $n$-butane in a miniature supersonic jet can lead selectively to simple translational-rotational relaxation, to rotational isomerization without condensation, or to condensation into large clusters depending upon expansion conditions. Expansion through a small thin plate nozzle brings about a rapid temperature drop with a minimum of collisions and induces neither conformational cooling nor observable cluster formation if carrier gas is absent. Expansions through conical nozzles are associated with less precipitous density and temperature drops, with a far more highly collimated jet, and, accordingly, with many more collisions before the terminal Mach number is reached. The cooling of pure butane by such expansions has not yet been seen to generate clusters but it has yielded substantial conversion of gauche to trans rotamers. Inclusion of enough helium or neon leads to considerable cluster formation with either type of nozzle. We conclude, then, that fast cooling, if sufficiently extreme, suffices to produce clusters while slow cooling, accompanied by numerous collisions, is needed for rotational isomerization of $n$-butane. Apparently $n$ butane, with a barrier of about $2.7 \mathrm{kcal} / \mathrm{mol},{ }^{5,13}$ is appreciably less free to isomerize than its close relative 1, 2-difluoroethane, whose barrier is $2.0 \mathrm{kcal} / \mathrm{mol}^{8}$ (in each case is cited the barrier to isomerization of the high energy form). Difluoroethane exhibited marked isomerization upon expansion from 400 Torr through a thin plate nozzle only $40 \mu \mathrm{m}$ in diam. ${ }^{8}$

Studies of nozzle-generated conformational cooling have several potentially interesting aspects, the full exploitation of which has not yet been realized. For one thing, it could, in principle, lead to a more complete characterization of the steps in the kinetic pathway for isomerization than is yet available. An ultrasonic relaxation experiment ${ }^{14}$ has measured the rate constant for butane, as a function of temperature, for the dense fluid where the unimolecular step is rate limiting. In nozzle expansions, the conversion is ultimately arrested by the slowness of the second-order activation step.

Another application is in structural chemistry. Although $n$-butane is the prototype example exhibiting trans-gauche isomerization, it has not yet been possible to determine, separately, the structure parameters of the two conformers. The best experimental information available ${ }^{7}$ consists of average bond lengths and bond angles for the trans-gauche gas phase mixtures. In principle, it is now possible to enrich the trans conformation greatly, to determine the trans structure itself by electron diffraction, and to infer the gauche parameters by deconvolution of the intensities for the mixture.

\section{ACKNOWLEDGMENTS}

This research was supported by a grant from the National Science Foundation. We thank M. Shetter, E. J. Valente, T. Trull, and Anding Jin for their assistance in the laboratory. We gratefully acknowledge a generous allocation of computing time from the University of Michigan Computing Center.

${ }^{1}$ See, for example, O. Bastiansen, K. Kveseth, and H. Mollendal, in Large Amplitude Motions in Molecules I, Topics in Current Chemistry (Springer, New York, 1979), Vol. 81; O. Bastiansen, H. M. Seip, and J. E. Boggs, in Perspectives in Structural Chemistry, edited by J. D. Dunitz and J. A. Ibers (Wiley, New York, 1971), Vol. IV.

${ }^{2}$ For example, K. Hagen and K. Hedberg, J. Am. Chem. Soc. 95, 4796 (1973).

${ }^{3}$ See discussion by R. L. Hilderbrandt, in Molecular Structure by Diffraction Methods, edited by G. A. Sim and L. E. Sutton (Specialist Periodical Report, Chemical Society, London, 1974), Vol. 3, p. 41.

${ }^{4}$ P. Felder and Hs. H. Gunthard, J. Mol, Struct. 60, 297 (1980).

${ }^{5}$ D. A. C. Compton, S. Montero, and W. F. Murphy, J. Phys. Chem. 84, 3587 (1980).

${ }^{6} \mathrm{~J}$. D. Lambert, Vibrational and Rotational Relaxation in Gases, (Clarendon, Oxford, 1977), p. 65: R. Holmes, G. R. Jones, and R. Lawrence, Trans. Faraday Soc. 62, 46 (1966).

${ }^{7}$ W. F. Bradford, S. Fitzwater, and L. S. Bartell, J. Mol. Struct. 38, 185 (1977).

${ }^{8}$ R. K. Heenan and L. S. Bartell, J. Chem. Phys. 78, 1265 (1983).

${ }^{9}$ R. K. Heenan, E. J. Valente, and L. S. Bartell, J. Chem. Phys. 78, 243 (1983).

${ }^{10}$ L. S. Bartell, R. K. Heenan, and M. Nagashima, J. Chem. Phys. 78, 236 (1983).

${ }^{11}$ L. S. Bartell, M. A. Kacner, and S. R. Goates, J. Chem. Phys. 75, 2730 (1981).

${ }^{12}$ P. Pulay, R. Mawhorter, D. A. Kohl, and M. Fink, J. Chem. Phys, (submitted).

${ }^{13}$ For a review of experimental and theoretical values, see $\mathrm{N}$. L. Allinger and S. Propeta, Jr. , J. Comput. Chem. 1, 181 (1980).

${ }^{14} \mathrm{~J}$. E. Piercy and M. G. S. Rao, J. Chem. Phys. 46, 3951 (1967). 